\title{
Histone modifications in fatty acid synthase modulated by carbohydrate responsive element binding protein are associated with non-alcoholic fatty liver disease
}

\author{
CAN CAI, HUIHONG YU, GUANGMING HUANG, XUAN DU, XIAOQING YU, YOUPING ZHOU and WEI SHEN \\ Department of Gastroenterology, 2nd Affiliated Hospital of Chongqing Medical University, Chongqing 400010, P.R. China
}

Received April 28, 2017; Accepted April 26, 2018

DOI: $10.3892 /$ ijmm.2018.3702

\begin{abstract}
Non-alcoholic fatty liver disease (NAFLD) is a manifestation of metabolic syndrome in the liver and is closely associated with diabetes; however, its pathogenesis remains to be elucidated. Carbohydrate responsive element binding protein (ChREBP), the hub of glucolipid metabolism, regulates the induction of fatty acid synthase (FASN), the key enzyme of de novo lipogenesis, by directly binding to carbohydrate response element (ChoRE) in its promoter. Investigations of histone modifications on NAFLD remain in their infancy. In the present study, by using ChIP, the association between histone modifications and FASN transcription was investigated and histone modifications in FASN modulated by ChREBP were measured. It was demonstrated that ChREBP induced FASN ChREBP-ChoRE binding to accelerate the expression of FASN, leading to hepatocellular steatosis by facilitating $\mathrm{H} 3$ and $\mathrm{H} 4$ acetylation, $\mathrm{H} 3 \mathrm{~K} 4$ trimethylation and the phosphorylation of H3S10, but inhibiting the trimethylation of H3K9 and H4K20 in FASN promoter regions of HepG2 and L02 cells. It was also found that ChREBP-ChoRE binding of FASN relied on histone acetylation and that the transcriptional activity of ChREBP on FASN is required, based on the premise that histone acetylation causes conformational changes in FASN chromatin. This indicated histone acetylation as a crucial mechanism involved in the transcription of FASN modulated by ChREBP.
\end{abstract}

Correspondence to: Professor Wei Shen, Department of Gastroenterology, 2nd Affiliated Hospital of Chongqing Medical University, 76 Linjiang Road, Yuzhong, Chongqing 400010, P.R. China

E-mail: shenwei315@126.com

Abbreviations: NAFLD, non-alcoholic fatty liver disease; ChREBP, carbohydrate responsive element binding protein; FASN, fatty acid synthase; HAT, histone acetyltransferase; HDAC, histone deacetylase; ChoRE, carbohydrate response element; ChIP, chromatin immunoprecipitation

Key words: histone modifications, fatty acid synthase, carbohydrate responsive element binding protein, hepatocellular steatosis, non-alcoholic fatty liver disease
Consequently, the present study provides further insight into the pathophysiology and a novel therapeutic potential of NAFLD based on epigenetic mechanisms.

\section{Introduction}

Non-alcoholic fatty liver disease (NAFLD) is a manifestation of metabolic syndrome in the liver and is characterized by ectopic lipid accumulation in hepatocytes without excess alcohol intake, which encompasses a spectrum from simple steatosis to steatohepatitis, cirrhosis and hepatic carcinoma (1). Over the last decade, NAFLD has emerged as one of the most common chronic liver diseases, with a prevalence of $20-30 \%$ in the general population in China (2), however, its pathogenesis remains to be elucidated. NAFLD is closely associated with diabetes. Abnormal glucolipid metabolism can result in fatty degeneration in hepatocytes $(3,4)$. Consequently, examination of the mechanism underlying abnormal glucolipid metabolism is particularly important for elucidating the pathogenesis of NAFLD and identifying novel therapeutic approaches for its treatment.

Carbohydrate responsive element binding protein (ChREBP) has been recognized as a key transcription factor of lipid synthesis, which is activated by high glucose, and its target genes involve glycolytic enzymes, gluconeogenic enzymes and lipid synthases (5-9). In response to an excessive glucose influx in hepatocytes, the expression and transcriptional activity of ChREBP are increased via liver $\mathrm{X}$ receptor (LXR) and phosphopentose pathways, respectively, which induce the transcription and expression of the downstream target gene fatty acid synthase (FASN), the key enzyme of lipid de novo synthesis, promoting the conversion of glucose to lipid (10-12). Therefore, ChREBP is considered a hub for glucolipid metabolism and a crucial regulator of de novo lipogenesis (11,13-15). In hepatocytes, the overexpression of FASN caused by excessive activation of ChREBP can result in hepatic steatosis and further progression of NAFLD (16-18).

Eukaryotic gene transcription is a highly regulated process, wherein the transformation of the chromatin structure is essential. All types of biological molecular signals ultimately exert their effect on the chromatin structure through various signaling pathways. However, chromatin is intrinsically positioned in a condensed and closed state, which limits various biological processes that require DNA as a template; therefore, 
the binding of any signaling molecules, including transcription factors, to the transcriptional regulatory elements of a target gene require accompanied changes in the local chromatin structure to activate or inhibit gene transcription. Due to the high stability of genes, signaling molecules generally affect gene alternative expression by altering the structure of chromatin without altering the DNA sequence. Epigenetics describes the reversible changes in gene activity, which can be inherited through generations and cell divisions, and eventually affects the phenotype through alterations to the chromatin structure and DNA methylation to enable the fine tuning of gene transcription without variation in the DNA sequence; this is vital in the differentiation of cells and the development of specific diseases $(19,20)$. Previous advances in the understanding of epigenetics and its prevalence as a contributor to the development of metabolic syndrome, including obesity and diabetes, have resulted in a marked increase in interest (21-24). As an important component of epigenetics, histone modification requires the cooperation of various enzymes, co-activators and transcriptional factors, including histone acetyltransferase (HAT), histone deacetylase (HDAC) and p300 $(25,26)$. Through histone modification, the interaction between histones and DNA, and between the promoter region of target genes and transcription factors, can be affected such that the condensation status of the chromatin can be altered to facilitate or inhibit gene transcription. ChREBP regulates the selective transcription of FASN by directly binding to the carbohydrate response element (ChoRE) found in the FASN promoter. However, no histone modifications of FASN modulated by ChREBP have been reported; therefore, their investigation is important in understanding glucolipid metabolism disorders and abnormal lipid deposition in the liver.

In the present study, HepG2 and L02 cell lines were selected as model cell lines. First, the association between histone modifications and transcription of FASN upon stimulation with high glucose, or following its withdrawal, was examined. Second, the effect of ChREBP on histone modifications in FASN promoter regions was determined using RNA interference and overexpression approaches. Finally, the effect of the histone acetylation pattern of FASN on hepatocellular lipogenesis was examined using the HAT inhibitor. The present study aimed to provide further insight into the pathophysiology of hepatic steatosis induced by high glucose and to identify the patterns of FASN histone modifications as a novel strategy for NAFLD therapy based on epigenetic mechanisms.

\section{Materials and methods}

Cell culture and reagents. The human normal liver cell line (L02 cells) and the hepatocellular carcinoma cell line (HepG2 cells) were obtained from the Cell Bank of the Institute of Biochemistry and Cell Biology (Shanghai, China). The HepG2 cells were cultured in Dulbecco's modified Eagle's medium (Hyclone; GE Healthcare, Life Sciences, Logan, UT, USA) containing $10 \%$ fetal bovine serum (FBS; Hyclone; GE Healthcare Life Sciences). The L02 cells were cultured in RPMI-1640 medium (Gibco; Thermo Fisher Scientific, Inc., Waltham, MA, USA) containing 10\% FBS (Hyclone; GE Healthcare Life Sciences). Both of the cell lines were cultured at $37^{\circ} \mathrm{C}$ with $5 \% \mathrm{CO}_{2}$ in a humidified incubator. The optimal glucose concentrations for HepG2 or L02 were analyzed using CCK8 assay kit (Applygen Technologies, Inc., Beijing, China), which revealed that 50 and $30 \mathrm{mM}$ were the optimal glucose concentrations for the HepG2 and L02 cells, respectively. Garcinol, an HAT inhibitor, was obtained from Cayman Chemical Company (Ann Arbor, MI, USA) and the treatment was $5 \mu \mathrm{M}$ in $5 \mathrm{ml}$ cell culture medium for $24 \mathrm{~h}$ as previously described (27).

Hepatic triglyceride (TG) assay. To determine the content of TG in the hepatocytes, the level of TG was assayed using a TG assay kit (Applygen Technologies, Inc.) according to the manufacturer's recommended protocol.

Oil Red $O$ staining. The cultured cells were rinsed with PBS three times for 5 min each and fixed with $4 \%$ paraformaldehyde for $30 \mathrm{~min}$ at $37^{\circ} \mathrm{C}$. The fixed cells were washed with PBS and stained with Oil Red O (Sigma-Aldrich; Merck KGaA, Darmstadt, Germany) for 10-15 min at room temperature. Subsequently, the stained cells were washed with PBS, counterstained with hematoxylin for 6-10 sec, and then rinsed with distilled water. Representative photomicrographs were captured at higher magnifications using a system incorporated into an optical microscope.

RNA isolation and reverse transcription-quantitative polymerase chain reaction $(R T-q P C R)$ analysis. Total RNA from the hepatocytes was extracted using TRIzol reagent (Invitrogen; Thermo Fisher Scientific, Inc.) and was reverse transcribed using the RevertAid First Strand cDNA Synthesis kit (Fermentas; Thermo Fisher Scientific, Inc.) according to the manufacturer's protocol. The qPCR analysis was performed using the KAPA SYBR ${ }^{\circledR}$ FAST qPCR kit Mater Mix ABI Prism ${ }^{\mathrm{TM}}$ (Kapa Biosystems, Boston, MA, USA) with the amplifying primer pairs: FASN, forward 5'-TTCTACGGC TCCACGCTCTTCC-3' and reverse 5'-GAAGAGTCTTCG TCAGCCAGGA-3'; and ChREBP, forward 5'-GTCGGAGAA CTTGCAGGAGT-3' and reverse 5'-CCTTCCAGCGACGGT CATC-3'. $\beta$-actin, forward 5'-TGACGGTCAGGTCATCAC TATCGG-3' and reverse 5'-TTGATCTTCATGGTGATAGGA GCGA-3' served as an internal control. qPCR master mix was as follows: $10 \mu \mathrm{l} 2 \mathrm{X}$ Master Mix, $0.4 \mu \mathrm{l} 10 \mu \mathrm{m}$ forward primer, $0.4 \mu \mathrm{l} 10 \mu \mathrm{m}$ reverse primer, $20 \mathrm{ng}$ template cDNA, and moderate PCR-grade buffer, the total volume was $20 \mu 1$. The thermocycling steps were as follows: i) Enzyme activation: $95^{\circ} \mathrm{C}$ for $3 \mathrm{~min}$, followed by 40 cycles of ii) Denaturation: $95^{\circ} \mathrm{C}$ for $3 \mathrm{sec}$ and iii) Annealing: $60^{\circ} \mathrm{C}$ for $20 \mathrm{sec}$. All samples were run in triplicate. Gene expression was normalized against $\beta$-actin, and the relative mRNA expression was calculated using the $2^{-\triangle \Delta C \mathrm{q}}$ method (28).

Western blot analysis. Total protein of hepatocytes was extracted using Protein Extraction kit (Sigma-Aldrich; Merck $\mathrm{KGaA}$ ) according to the manufacturer's protocol. Briefly, $80 \mu \mathrm{g}$ protein lysates were separated by $5-15 \%$ sodium dodecyl sulfate-polyacrylamide gel electrophoresis and subsequently transferred onto polyvinylidene difluoride membranes. Following blocking with PBST containing 5\% fatty acid-free milk, the membranes were incubated overnight at $4^{\circ} \mathrm{C}$ with specific primary antibodies against ChREBP $(1: 1,000$; cat. 
no. ab157153; Abcam, Cambridge, MA, USA), FASN (1:200; cat. no. sc-20140; Santa Cruz Biotechnology, Inc., Dallas, TX, USA) or $\beta$-actin $(1: 1,000$; cat. no. SP124; EMD Millipore; Billerica, MA, USA) and the corresponding HRP-conjugated secondary antibodies (1:2,000; cat. no. ab6721; Abcam) at room temperature $\left(25^{\circ} \mathrm{C}\right)$ for $2 \mathrm{~h}$. The protein bands were visualized using ECL western blotting reagents. The band intensities were quantified using Quantity One-4.6.2 software (Bio-Rad Laboratories, Inc., Hercules, CA, USA).

Plasmids, small interfering RNA (siRNA) and transient transfection. ChREBP-specific siRNA (siRNA-ChREBP) and GV102-scramble (negative control) plasmids were purchased from Shanghai GenePharma Biotechnology (Shanghai, China). The target sequences of the siRNA-ChREBP and GV102-scramble were 5'-GCAGAAGACAGCTGAGTA CAT-3' and 5'-GTTCTCCGAACGTGTCACGT-3', respectively. Cells at $85-90 \%$ confluence were transfected with either siRNA-ChREBP or siRNA-scramble using PolyJet in vitro DNA transfection reagent (SignaGen Laboratories, Rockville, MD, USA). The transfection efficiency, which was measured by quantifying the number of EGFP-positive cells using a fluorescence microscope, was $\sim 70-80 \%$. For the overexpression experiments, a plasmid expressing human wild-type ChREBP (GenBank accession no. NG_009307) was also purchased from GenePharma Biotechnology. The cells were transfected with the ChREBP plasmid or pEX3-scramble (negative control) using PolyJet, as described above.

Chromatin immunoprecipitation (ChIP) assay. The cells were cross-linked with $1 \%$ formaldehyde for $10 \mathrm{~min}$ at $37^{\circ} \mathrm{C}$, and crude nuclei were purified using a previously described protocol (27). The crude nuclei were sonicated to produce chromatin fragments of $\sim 500 \mathrm{bp}$, as determined by agarose gel electrophoresis. For immunoprecipitation, the antibodies used in the ChIP assay were as follows: Anti-acetyl-histone H3 (cat. no. 06-599; EMD Millipore), anti-acetyl-histone H4 (cat. no. 06-866; EMD Millipore), anti-phospho-histone H3 serine 10 (H3S10, cat. no. 04-817; EMD Millipore), and anti-trimethyl-histone H4 lysine 20 (cat. no. 07-463; EMD Millipore), in addition to anti-trimethyl-histone $\mathrm{H} 3$ lysine 4 (H3K4, cat. no. ab12209; Abcam), anti-trimethyl-histone $\mathrm{H} 3$ lysine 9 (H3K9, cat. no. ab8898; Abcam) and anti-ChREBP (cat. no. ab157153; Abcam). For each ChIP assay, $2.5 \mu \mathrm{g}$ of antibody was added and the samples were incubated overnight at $4^{\circ} \mathrm{C}$. The antibody-chromatin complexes were precipitated by overnight incubation with Protein A Agarose/Salmon Sperm DNA at $4{ }^{\circ} \mathrm{C}$. The DNA-protein crosslinks were reversed by incubation at $65^{\circ} \mathrm{C}$ for $4 \mathrm{~h}$, followed by proteinase $\mathrm{K}$ treatment. DNA was recovered using a purification column (Qiagen $\mathrm{GmbH}$, Hilden, Germany). The ChIP and input DNA samples were quantified by RT-qPCR analysis, and each assay was performed in triplicate. For the FASN regulatory regions, the following were selected: ChoRE (forward 5'-CTTTGTCCGCACCACACC AGG-3' and reverse 5'-AGACCCGAGACGGACGTCCAC-3'), -2 kb (forward 5'-TGGGAACACGATGGGAGAAC-3' and reverse 5'- ATCGTGGGACCTTTGCAGC-3') upstream from the transcription start site (TSS), promoter (forward 5'-GAT GGCCGCGGTTTAAATAGC-3' and reverse 5'-AGAGAC GGCAGCGGC-3'), and exon2 (forward 5'-CGGGAAGCT
GCCAGAGT-3' and reverse 5'-TTCTGGGACAACCTCAT CGG-3').

Statistical analysis. SPSS software (version 17.0; SPSS, Inc., Chicago, IL, USA) was used to analyze the data which are expressed as the mean \pm standard deviation. All experiments were performed in triplicate. Differences among groups were determined using the Student Newman Keuls test. $\mathrm{P}<0.05$ was considered to indicate a statistically significant difference.

\section{Results}

Enhancement of lipogenesis in hepatocytes induced by high glucose. The HepG2 and L02 cells were incubated with high glucose for 0,24 and $48 \mathrm{~h}$. Western blot analysis revealed that high glucose significantly increased the protein expression of FASN in the HepG2 and L02 cells in a time-dependent manner (Fig. 1A). Following stimulation of the HepG2 and L02 cells with high glucose for $48 \mathrm{~h}$ in, the culture medium containing high glucose was replaced with normal medium, termed 'glucose removal', which was followed by the measurement of FASN protein levels using western blot analyses at 0 , 24 and 48 h later. As shown in Fig. 1B, when high glucose was removed for $48 \mathrm{~h}$ in the HepG2 and L02 cells, the protein level of FASN was markedly decreased, compared with the level at $0 \mathrm{~h}$ glucose removal, although no significant difference was found between 24 and $0 \mathrm{~h}$ glucose removal. As shown in Fig. 1C, the TG contents of the HepG2 and L02 cells were increased over time. To further substantiate this data, the levels of fat droplets were examined in HepG2 and L02 cells using Oil Red O staining analysis. The high glucose treatment induced fat droplets in the HepG2 and L02 cells, with the same pattern observed using western blot analyses and the TG content assay (Fig. 1D). However, in the two cell lines at $48 \mathrm{~h}$ glucose removal, the fat droplets were decreased compared with those at $0 \mathrm{~h}$ glucose removal, although no significant difference was found between 24 and $0 \mathrm{~h}$ glucose removal (Fig. 1E). Taken together, these results suggested that high glucose induced the enhancement of lipogenesis in hepatocytes.

Association between histone modifications and transcription of FASN in the presence of high glucose and following its removal. Following the observation of the increased lipogenesis potential of hepatocyte lines treated with high glucose, the time spectrum of the mRNA expression of FASN was investigated in the presence of high glucose or following its removal using RT-qPCR analysis. The mRNA expression levels of FASN in the two hepatocyte lines in the presence of high glucose are shown in Fig. 2A. The mRNA expression of FASN was temporally increased at $1 \mathrm{~h}$, compared with that at $0 \mathrm{~h}$, however, it returned to baseline over the subsequent hours. When the HepG2 and L02 cells were incubated in high glucose for 12 and $8 \mathrm{~h}$, respectively, the mRNA expression of FASN was gradually and stably increased with time. Following high glucose removal, the increasing mRNA level of FASN was significantly decreased with time in the HepG2 and L02 cells (Fig. 2A). All differences were of statistical significance. These experiments were used to determine suitable time-points for the following ChIP experiments. 
A
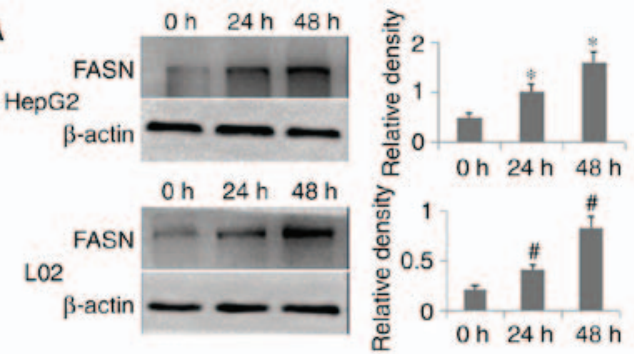

B
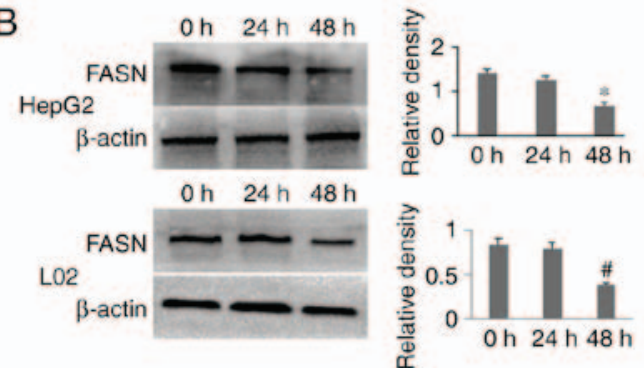

C

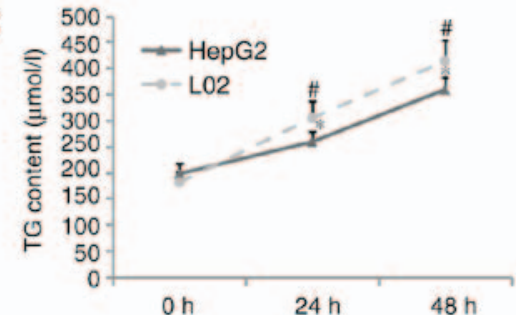

D

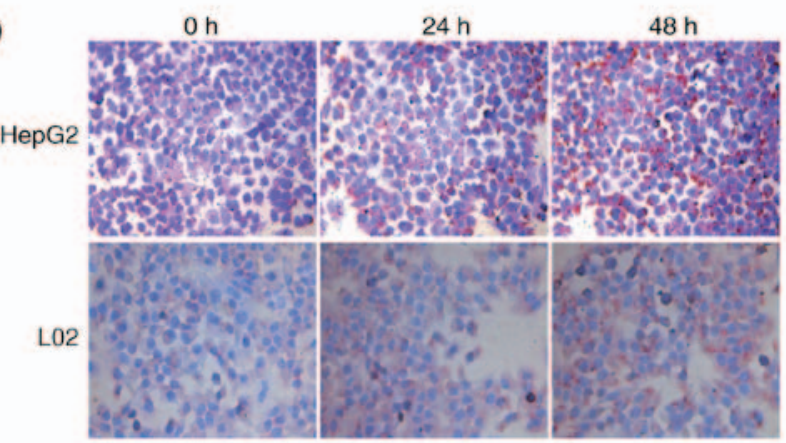

E

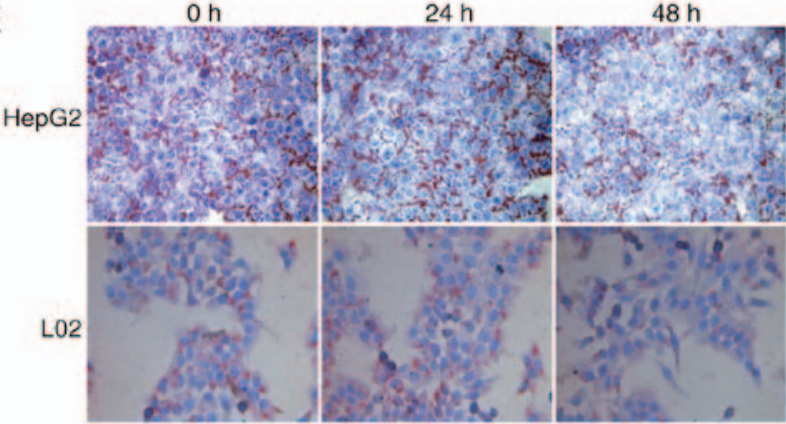

$\mathrm{Oh}$

$24 \mathrm{~h}$

$48 \mathrm{~h}$

Figure 1. Enhancement of lipogenesis in hepatocytes induced by high glucose. Protein levels of FASN in HepG2 and L02 cells under (A) glucose treatment or (B) following glucose removal were determined using western blot analyses; $\beta$-actin was used as a loading control. (C) TG contents were measured using a TG assay kit, and fat droplets were evaluated by Oil Red O staining in HepG2 and L02 cells under (D) glucose treatment or (E) following glucose removal. Magnification, $\mathrm{x} 200$. ${ }^{*} \mathrm{P}<0.05$, vs. $0 \mathrm{~h}$ in HepG2; ${ }^{*} \mathrm{P}<0.05$, vs. $0 \mathrm{~h}$ in L02. Data are expressed as the mean \pm standard deviation from at least three independent experiments. FASN; fatty acid synthase; TG, triglyceride; TSS, transcription start site.

To elucidate the underlying epigenetic modifications of FASN promoter regions under the stimulation of high glucose and following its withdrawal, ChIP experiments were performed in the hepatocyte lines. The promoter exhibits an extensive domain, which consists of a core promoter region and regulatory regions. The former is responsible for the transcription of genetic foundation levels, whereas regulatory regions are able to respond to different environmental factors or intracellular signals and then make the corresponding adjustments for the selective transcription of genes. Between the two domains, the core promoter is usually located within $-2,000 \mathrm{bp}$ from the TSS, and the promoter sequence of the human FASN gene exhibits high similarity with that of the rat (29) (Fig. 2B). With the ChoRE and core promoter locus as experimental groups, exon 2 and the $-2 \mathrm{~kb}$ locus were selected as controls.

Compared with $0 \mathrm{~h}$ exposure to high glucose, significant increases in $\mathrm{H} 3$ and $\mathrm{H} 4$ acetylation within the ChoRE, promoter and exon 2 regions were observed in the HepG2 cells following exposure to high glucose for $12 \mathrm{~h}$, and in the $\mathrm{L} 02$ cells following exposure to high glucose for $8 \mathrm{~h}$ (Fig. 2C and D). Higher degrees of phosphorylated H3S10 within the ChoRE, promoter and exon2 regions were also observed in the HepG2 and L02 cells, compared with that at $0 \mathrm{~h}$ (Fig. 2C and D). Depending on the site and the degree of histone methylation, modifications result in either gene activation or repression; increased H3K4 trimethylation was observed in the ChoRE, promoter and exon2 regions of the HepG2 and L02 cells following exposure to high glucose, compared with that at $0 \mathrm{~h}$ (Fig. 2C and D). By contrast, trimethylated H3K9 and H4K20 are repressive marks, and they function through different mechanisms (30). Significant decreases in H3K9 and H4K20 trimethylation were observed in the ChoRE, promoter and exon2 regions of the HepG2 and L02 cells following exposure to high glucose, compared with that at $0 \mathrm{~h}$ (Fig. 2C and D).

When high glucose was removed, as previously mentioned, for $1 \mathrm{~h}$, it was observed that the increasing ranges of $\mathrm{H} 3$ and $\mathrm{H} 4$ acetylation, $\mathrm{H} 3 \mathrm{~S} 10$ phosphorylation, and $\mathrm{H} 3 \mathrm{~K} 4$ trimethylation were decreased, whereas $\mathrm{H} 3 \mathrm{~K} 9$ trimethylation and $\mathrm{H} 4 \mathrm{~K} 20$ trimethylation were increased in the FASN promoter regions of the HepG2 and L02 cells, compared with the ranges at $0 \mathrm{~h}$ high glucose removal (Fig. 2E and F). Under high glucose treatment and following its removal, no changes in epigenetic marks were detected within $-2 \mathrm{~kb}$ in either hepatocytes, compared with the $0 \mathrm{~h}$ controls. According to these results, the levels of $\mathrm{H} 3$ and $\mathrm{H} 4$ acetylation, H3S10 phosphorylation and H3K4 trimethylation were increased, but the trimethylation levels of $\mathrm{H} 3 \mathrm{~K} 9$ and $\mathrm{H} 4 \mathrm{~K} 20$ were reduced in FASN under a high glucose stimulus, which resulted in activation of the transcription of FASN. Following the removal of glucose, the increasing levels of $\mathrm{H} 3$ and $\mathrm{H} 4$ acetylation, $\mathrm{H} 3 \mathrm{~S} 10$ phosphorylation and H3K4 trimethylation levels all declined, whereas the levels of H3K9 

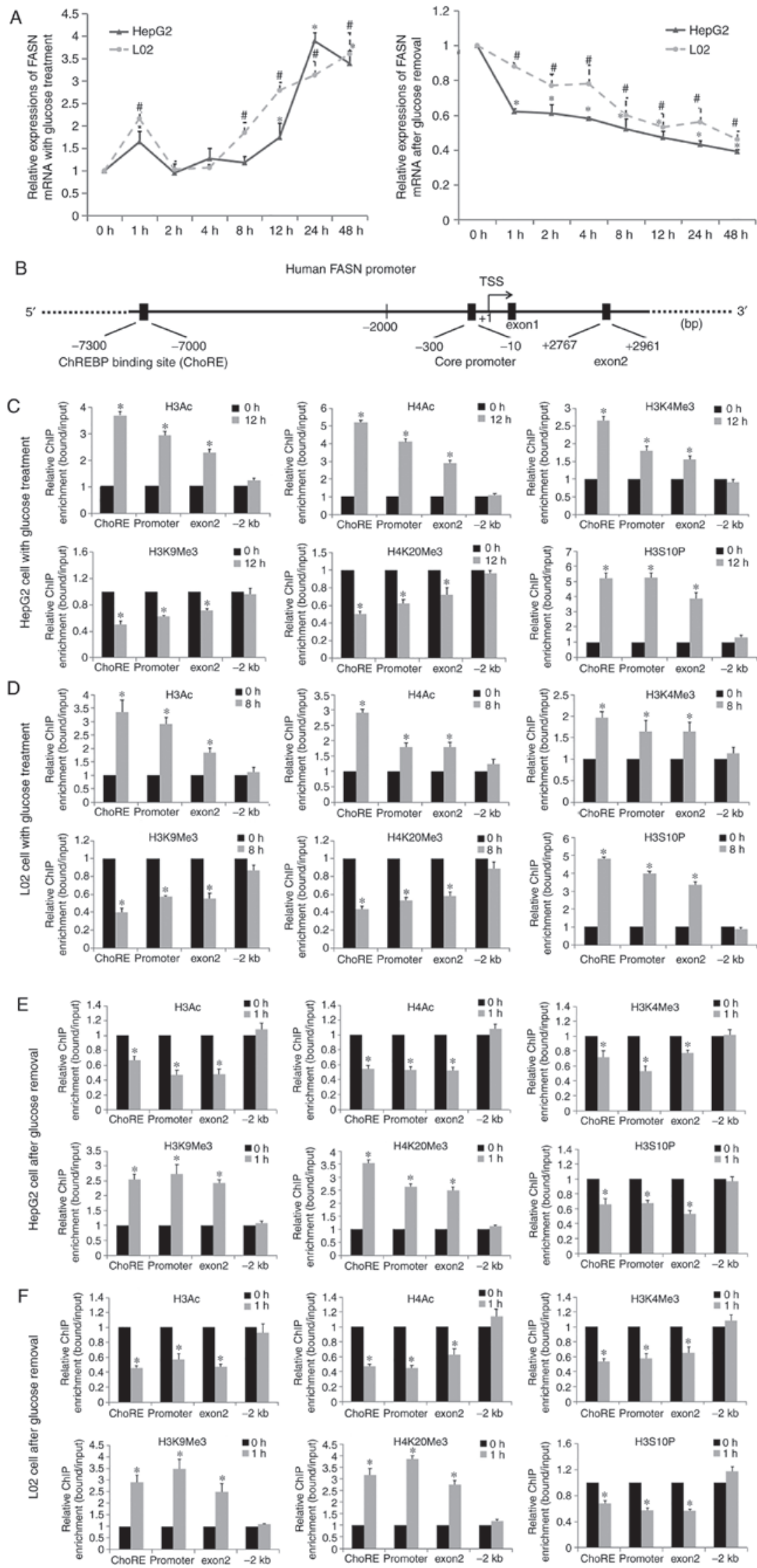

Figure 2. Association between histone modifications and transcription of FASN in the presence of a high glucose concentration and following its removal. Total RNA was extracted and used for reverse transcription-quantitative polymerase chain reaction analysis to examine the time mRNA expression of FASN in HepG2 and L02 cells (A) under glucose treatment or following removal; $\beta$-actin was used as an internal control; ${ }^{*} \mathrm{P}<0.05$, vs. $0 \mathrm{~h}$ in $\mathrm{HepG} 2$; ${ }^{\mathrm{P}}<0.05$, vs. $0 \mathrm{~h}$ in $\mathrm{L} 02$. (B) Analysis of FASN promoter regions around the TSS. Chromatin immunoprecipitation assays were used to examine acetylated $\mathrm{H} 3$ and $\mathrm{H} 4$, and trimethylated $\mathrm{H} 3 \mathrm{~K} 4$, $\mathrm{H} 3 \mathrm{~K} 9$ and H4K20, and phosphorylated H3S10 occupancy in the FASN ChoRE, promoter, exon2, and -2 kb regions of (C) HepG2 and (D) L02 cells treated with glucose, and (E) HepG2 and (F) L02 cells following gluocse removal. Values at time $0 \mathrm{~h}$ were set as $1 ;{ }^{*} \mathrm{P}<0.05$, vs. $0 \mathrm{~h}$ in HepG2 or L02. Data are expressed as the mean \pm standard deviation from at least three independent experiments. FASN; fatty acid synthase; ChoRE, carbohydrate response element; ChIP, chromatin immunoprecipitation. 
and H4K20 trimethylation increased in FASN, which induced a decrease in the increased transcription of FASN.

Involvement of ChREBP in histone modifications at the FASN promoter regions. Subsequently, the present study attempted to elucidate the mechanisms underlying histone modifications at the FASN promoter regions in the hepatocyte lines. ChREBP is the main molecule activated by the glucose signaling pathway and modulates the induction of FASN by directly binding to ChoRE found in the gene promoter. To determine the effect of ChREBP on the epigenetic modifications at the FASN promoter regions of the hepatocyte lines, the protein expression of ChREBP with high glucose incubation or its removal was evaluated using western blot analysis. As shown in Fig. 3A, the protein expression of ChREBP was distinctly enhanced with the increase in high glucose incubation time, which was accompanied by elevated intracellular lipogenesis, as mentioned above. However, when high glucose was removed for $48 \mathrm{~h}$ in the HepG2 and L02 cells, the protein level of ChREBP was markedly decreased compared with that at $0 \mathrm{~h}$ glucose removal, although no significant difference was found between 24 and $0 \mathrm{~h}$ glucose removal (Fig. 3B).

ChIP assays were also performed to examine the time spectrum of the ChREBP-ChoRE binding. When stimulated with high glucose, the ChREBP-ChoRE binding in the HepG2 and L02 cell lines increased almost linearly (Fig. 3C), as did the mRNA expression of FASN. Following high glucose removal, it was found that the increasing ChREBP-ChoRE binding decreased linearly (Fig. 3C), and this finding was consistent with a change in the mRNA expression levels of FASN. These findings demonstrated that glucose affected the transcription of FASN by regulating the expression and transcriptional activity of ChREBP. ChREBP-ChoRE binding and the expression of FASN also had a positive association.

The present study also investigated the functional role of ChREBP in the establishment of histone modifications at the FASN promoter regions by specifically knocking down or overexpressing endogenous ChREBP in the HepG2 and L02 cells. Following $48 \mathrm{~h}$ transfection with siRNA-ChREBP, the protein expression levels of ChREBP and FASN were reduced in the HepG2 and L02 cells, compared with levels in the normal group (Fig. 3D). Furthermore, Oil Red O staining was used to examine the fat droplet levels in the three groups, with no difference found between them (Fig. 3E). By contrast, the protein levels of ChREBP and FASN were markedly increased in the HepG2 and L02 cells compared with those in the normal group following 48-h transfection in the ChREBP-overexpression group (Fig. 3F). Using Oil Red O staining, it appeared that the degree of lipid accumulation was enhanced in the ChREBP-overexpression group, compared with that in the normal group, which was consistent with the protein expression of ChREBP and FASN between the two groups (Fig. 3G). The effect of the downregulation of ChREBP on histone modifications of FASN was then determined, and the HepG2 or L02 cells were divided into four groups, including the normal control (cultured in medium), positive control (exposed to high glucose for 12 or $8 \mathrm{~h}$ ), transfection group (exposed to high glucose for 12 or $8 \mathrm{~h}$ following 48-h transfection with the siRNA-ChREBP plasmid) and negative control (exposed to high glucose for 12 or $8 \mathrm{~h}$ following 48-h transfection with the GV102-scramble plasmid). The ChREBP-ChoRE binding of FASN was weaker in the transfection group, compared with that in the positive and negative controls in the two cell lines (Fig. 3H). As shown in Fig. 3I and J, FASN histone hypo-acetylation, H3S10 hypo-phosphorylation, H3K4 hypo-trimethylation, and H3K9 and H4K20 hyper-trimethylation at the ChoRE, promoter and exon2 regions were observed in the transfection group, compared with the positive and negative controls.

The present study also examined the effect of the upregulation of ChREBP on histone modifications of FASN, with hepatocytes also divided into four groups: Normal control (cultured in medium); positive control (exposed to high glucose for $48 \mathrm{~h}$ ); transfection group (transfected with the ChREBP-overexpression plasmid for $48 \mathrm{~h}$ ); and the negative control (transfected with the scramble plasmid for 48 h). Concomitantly, the ChREBP-ChoRE binding, histone acetylation, phosphorylation of $\mathrm{H} 3 \mathrm{~S} 10$ and trimethylation of $\mathrm{H} 3 \mathrm{~K} 4$ in the FASN promoter regions were increased in the transfection group, compared with the three control groups (Fig. 3H, K and L). By contrast, H3K9 and H4K20 trimethylation were decreased in the transfection group, compared with the three control groups (Fig. 3I and J). Regardless of whether transfection occurred with the siRNA-ChREBP or ChREBP-overexpression plasmid, there was no change in the epigenetic marks at $-2 \mathrm{~kb}$ among the four groups. It was concluded from the above results that ChREBP facilitated the acetylation of $\mathrm{H} 3$ and $\mathrm{H} 4$, trimethylation of $\mathrm{H} 3 \mathrm{~K} 4$ and phosphorylation of $\mathrm{H} 3 \mathrm{~S} 10$, and inhibited the trimethylation of $\mathrm{H} 3 \mathrm{~K} 9$ and $\mathrm{H} 4 \mathrm{~K} 20$ in the FASN promoter regions. This facilitated FASN ChREBP-ChoRE binding and regulated the transcription of FASN.

Hepatocellular steatosis is rescued by inhibiting histone acetylation. On observing the increase in histone acetylation of the FASN promoter regions in HepG2 and L02 cells under high glucose stimulation, the present study investigated whether this change affected the expression of FASN and hepatic lipid accumulation. The hepatocytes were divided into three groups: Normal control group (cultured in medium); glucose+garcinol group (treated with a high glucose/garcinol cocktail for $24 \mathrm{~h}$ ); and glucose group (treated with high glucose for $24 \mathrm{~h}$ ). The optimal garcinol concentration for HepG2 and L02 cells was $5 \mu \mathrm{M}$ as previously described (27). The high glucose/garcinol cocktail caused histone hypo-acetylation and decreased the ChREBP-ChoRE binding of FASN compared with the glucose group; however, this was increased compared with the normal group (Fig. 4A and B). Total RNA and protein were recovered for RT-qPCR and western blot analyses, respectively. As shown in Fig. 4C and D, the high glucose/garcinol cocktail failed to promote the protein and mRNA expression of FASN relative to the glucose group; however, it did increase the expression compared with the normal group in the two hepatocyte lines. The results of the TG content assay and Oil Red $\mathrm{O}$ staining were consistent with the expression of FASN (Fig. 4E and F). Finally, the protein expression of ChREBP in each group was examined. Western blot analysis revealed that the expression levels of ChREBP in the glucose+garcinol and glucose groups were almost the same but were higher compared with that in 
A
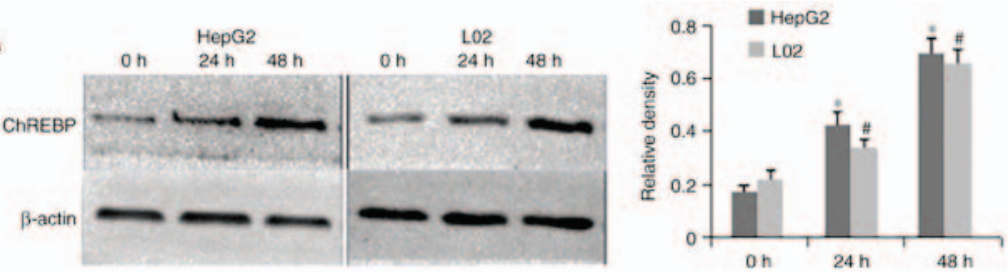

B
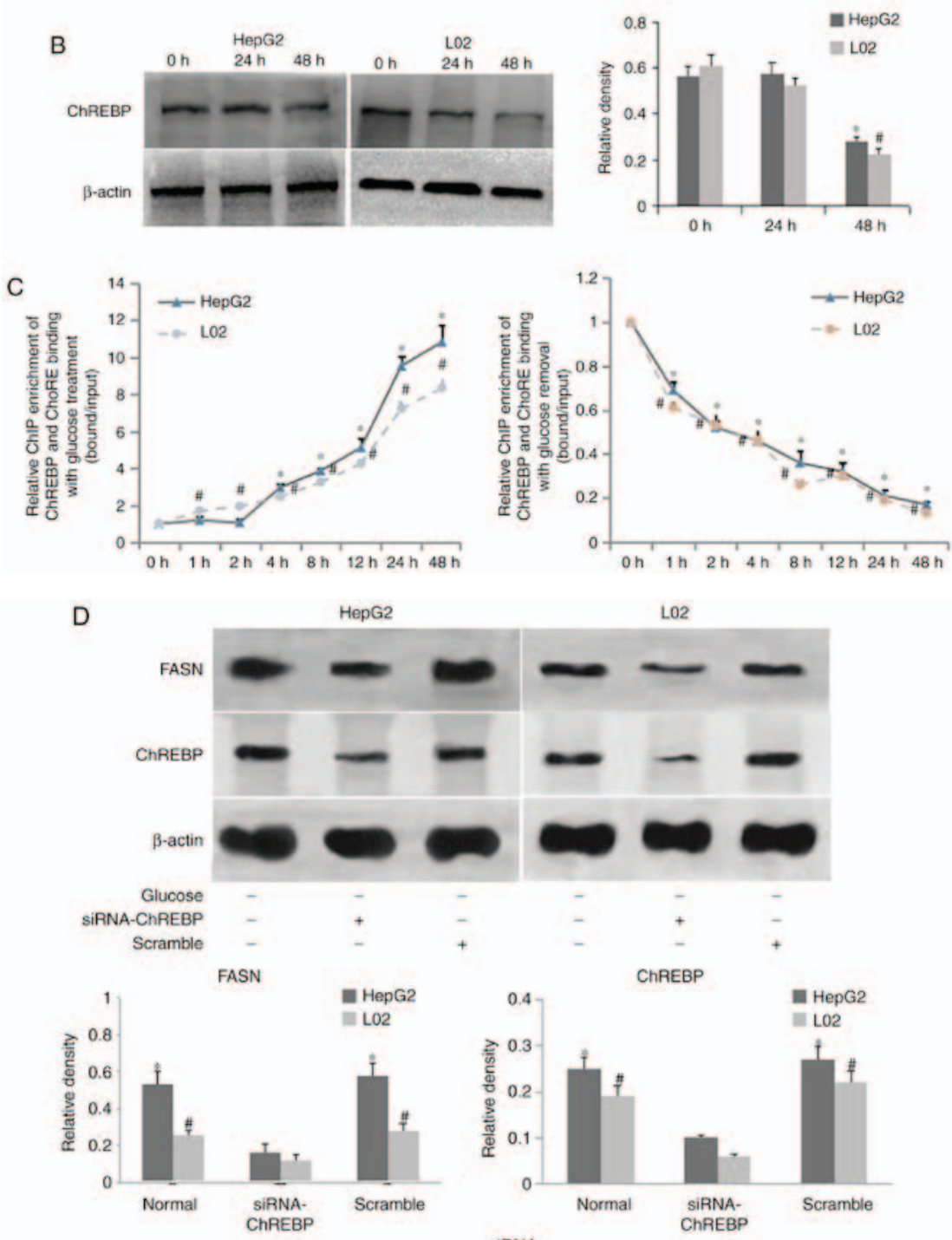

$E$

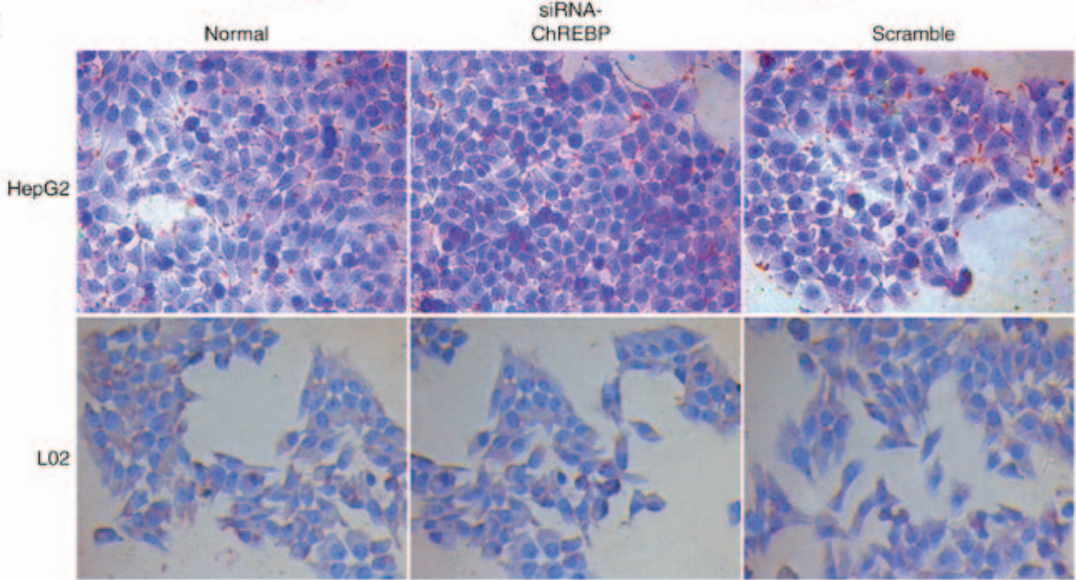

Figure 3. Involvement of ChREBP in histone modifications at the FASN promoter regions. Protein levels of ChREBP in HepG2 and L02 cells (A) with glucose treatment or (B) following glucose removal were determined using western blot analysis; $\beta$-actin was used as a loading control; " $\mathrm{P}<0.05$, vs. $0 \mathrm{~h}$ in HepG2; ${ }^{\#} \mathrm{P}<0.05$, vs. $0 \mathrm{~h}$ in L02. (C) ChIP-qPCR assays were used to examine the time spectrum of ChREBP-ChoRE binding in HepG2 and L02 cells with glucose treatment or removal; values at time $0 \mathrm{~h}$ were set as 1; "P $\mathrm{P}<0.05$, vs. $0 \mathrm{~h}$ in $\mathrm{HepG} 2 ;{ }^{*} \mathrm{P}<0.05$, vs. $0 \mathrm{~h}$ in L02. (D) Western blot analysis of protein levels of ChREBP and FASN, and (E) Oil Red O staining of fat droplets in HepG2 and L02 cells with siRNA-ChREBP plasmid transfection. 
$\mathrm{F}$

HepG2

L02
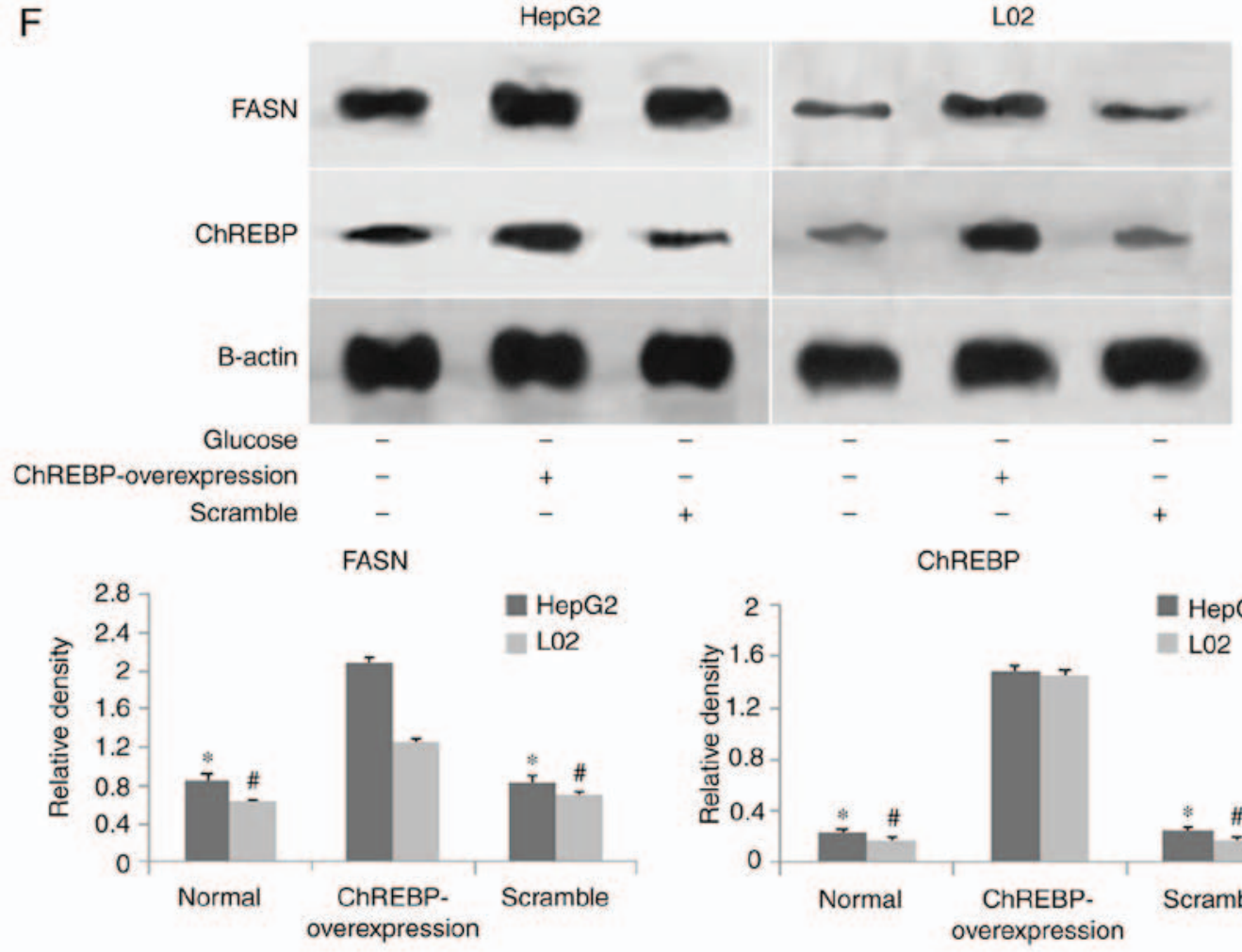

ChREBP

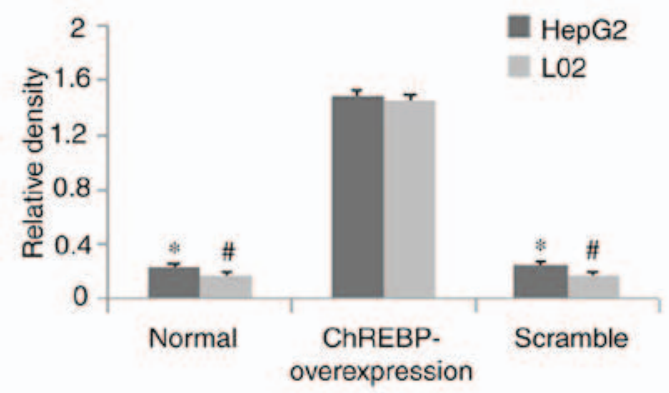

G

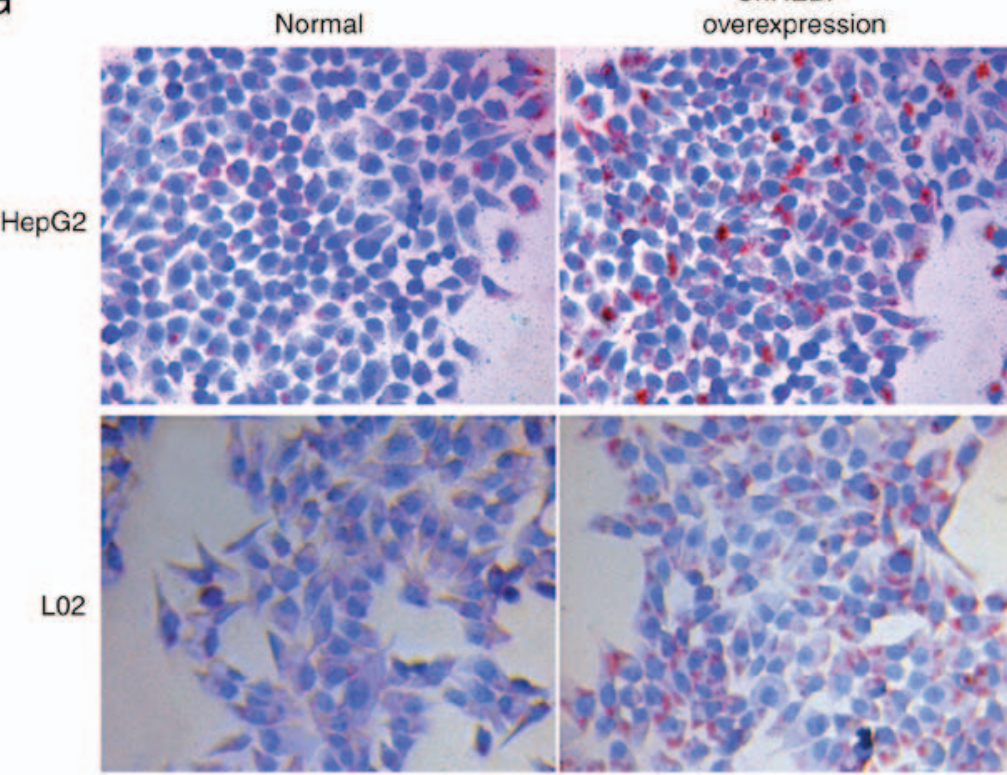

ChREBP-

overexpression

Scramble

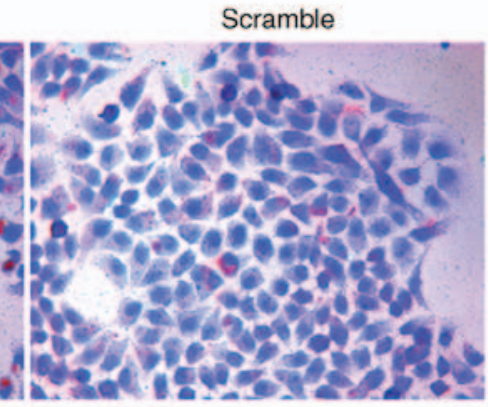

$\mathrm{H}$
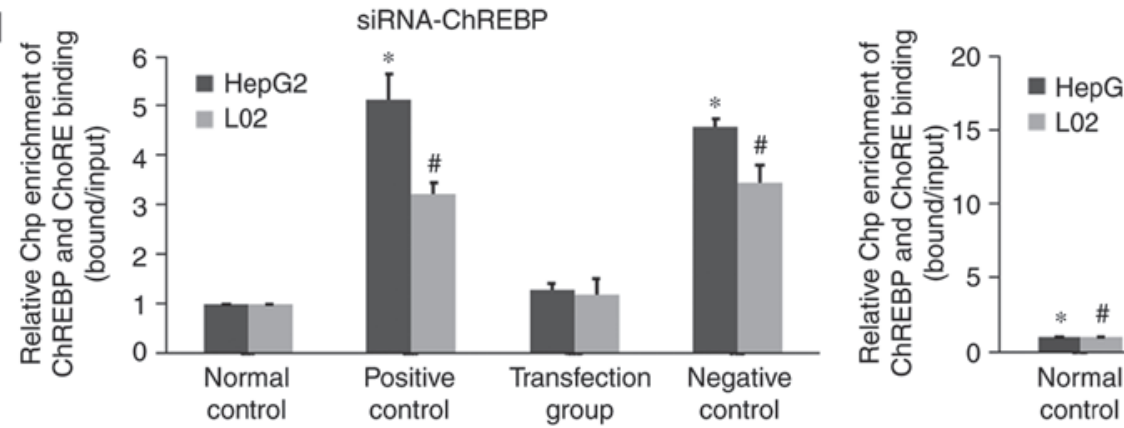

ChREBP-overexpression

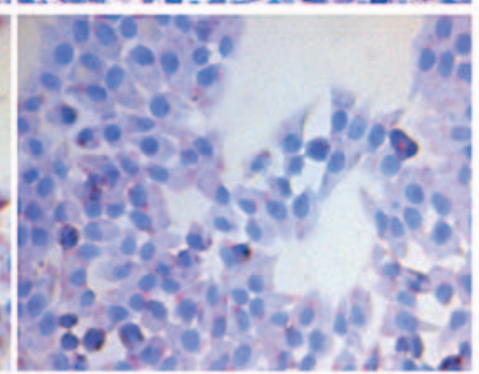

Figure 3. Continued. (F) Western blot analysis of protein levels of ChREBP and FASN, and (G) Oil Red O staining of fat droplets in HepG2 and L02 cells with ChREBP-overexpression plasmid transfection; $\beta$-actin was used as a loading control; " $\mathrm{P}<0.05$, vs. siRNA-ChREBP or ChREBP-overexpression group in HepG2; ${ }^{*} \mathrm{P}<0.05$, vs. siRNA-ChREBP or ChREBP-overexpression group in L02. Magnification, $\mathrm{x} 200$. (H) ChIP assays were used to investigate ChREBP-ChoRE binding; values of normal controls were set as $1,{ }^{*} \mathrm{P}<0.05$, vs. transfection group in HepG2; ${ }^{*} \mathrm{P}<0.05$, vs. transfection group in L02. 

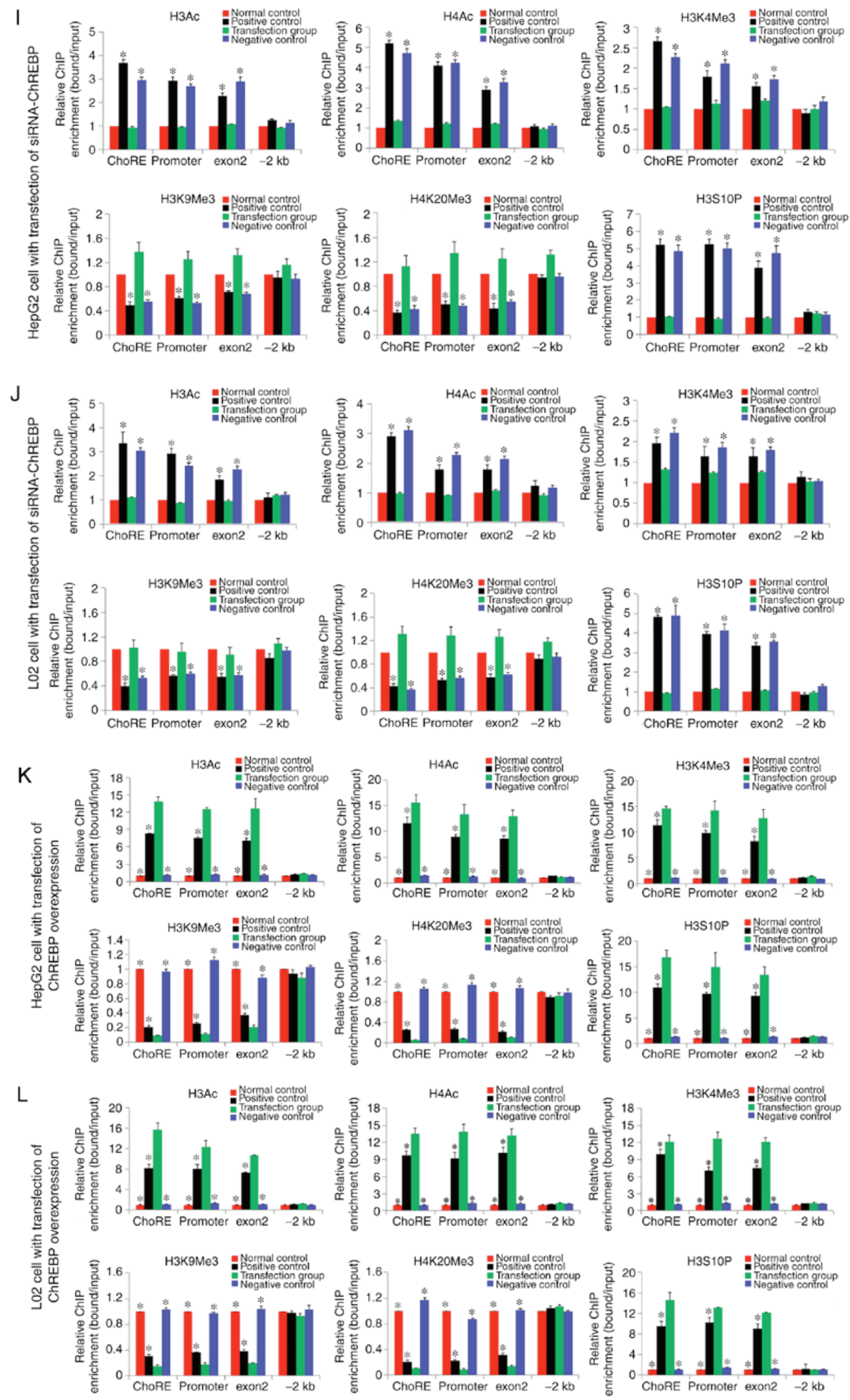

Figure 3. Continued. Acetylated H3 and H4, trimethylated H3K4, H3K9 and H4K20, and phosphorylated H3S10 occupancy in the FASN ChoRE, promoter, exon2, and -2 kb regions of (I) HepG2 and (J) L02 cells with transfection of siRNA-ChREBP and exposure to high glucose or (K) HepG2 and (L) L02 cells with transfection of ChREBP-overexpression, values of normal controls were set as $1,{ }^{*} \mathrm{P}<0.05$, vs. transfection group in HEepG2 or L02. Data are expressed as the mean \pm standard deviation from at least three independent experiments. FASN histone hypo-acetylation, H3S10 hypo-phosphorylation, H3K4 hypo-trimethylation, and $\mathrm{H} 3 \mathrm{~K} 9$ and $\mathrm{H} 4 \mathrm{~K} 20$ hyper-trimethylation at the ChoRE, promoter and exon2 regions were observed, ChREBP-ChoRE binding, histone acetylation, phosphorylation of $\mathrm{H} 3 \mathrm{~S} 10$, and trimethylation of $\mathrm{H} 3 \mathrm{~K} 4$ of the FASN promoter regions were increased, and trimethylation of $\mathrm{H} 3 \mathrm{~K} 9$ and $\mathrm{H} 4 \mathrm{~K} 20$ were decreased in the transfection group, compared with the three control groups. FASN; fatty acid synthase; ChoRE, ChoRE, carbohydrate response element; ChREBP, ChoRE binding protein; TSS, transcription start site; ChIP, chromatin immunoprecipitation; siRNA, small interfering RNA. 

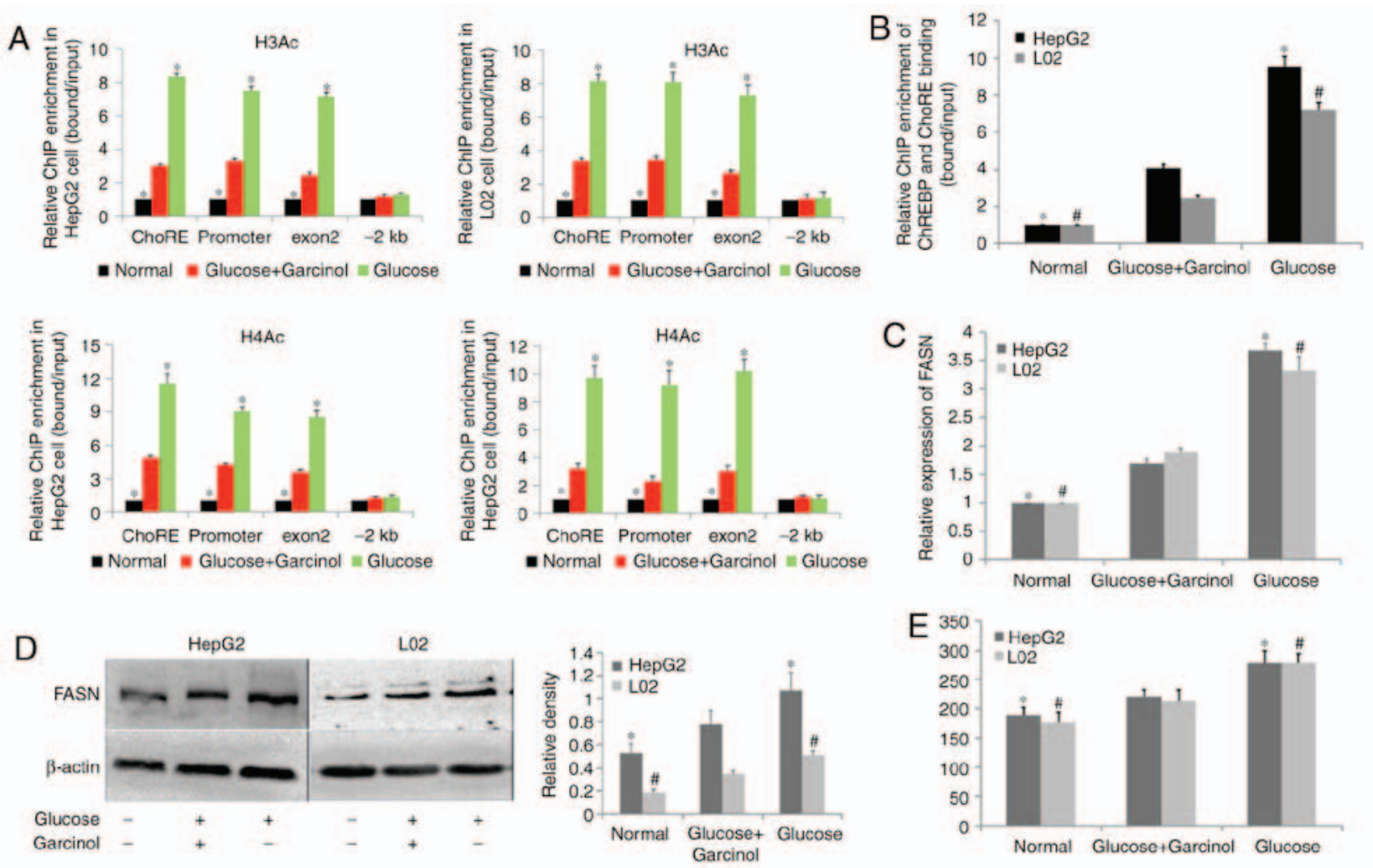

F

Normal

Glucose+Garcinol

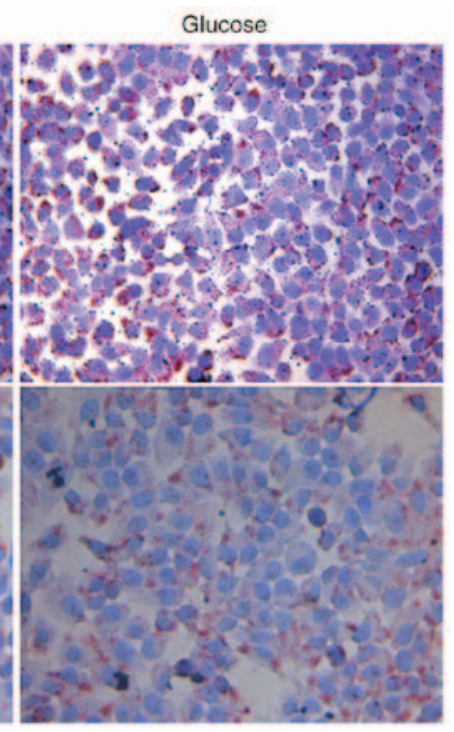

G
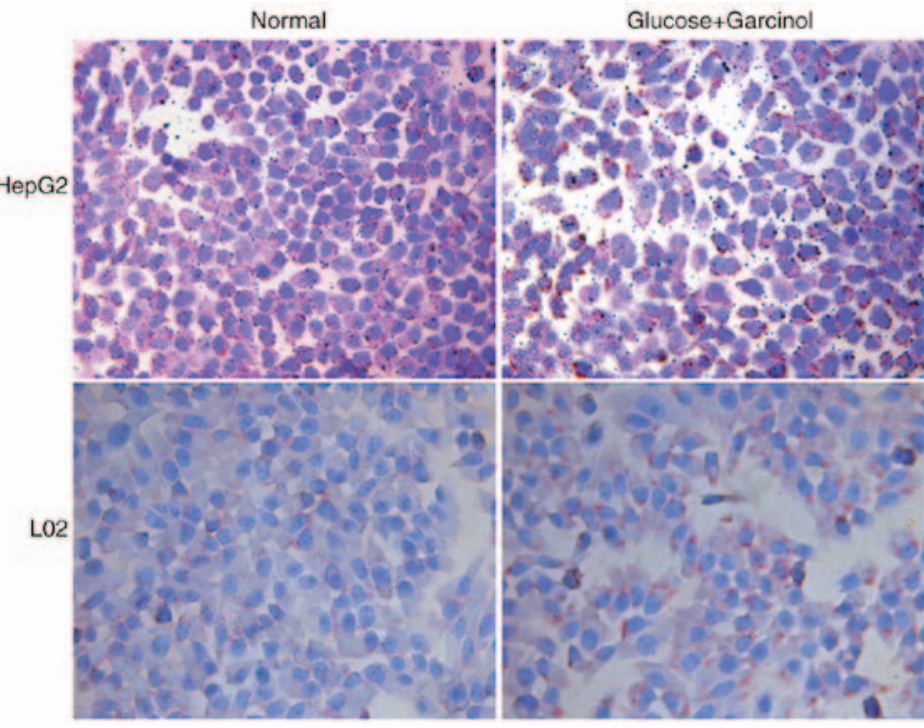

HepG2

L02
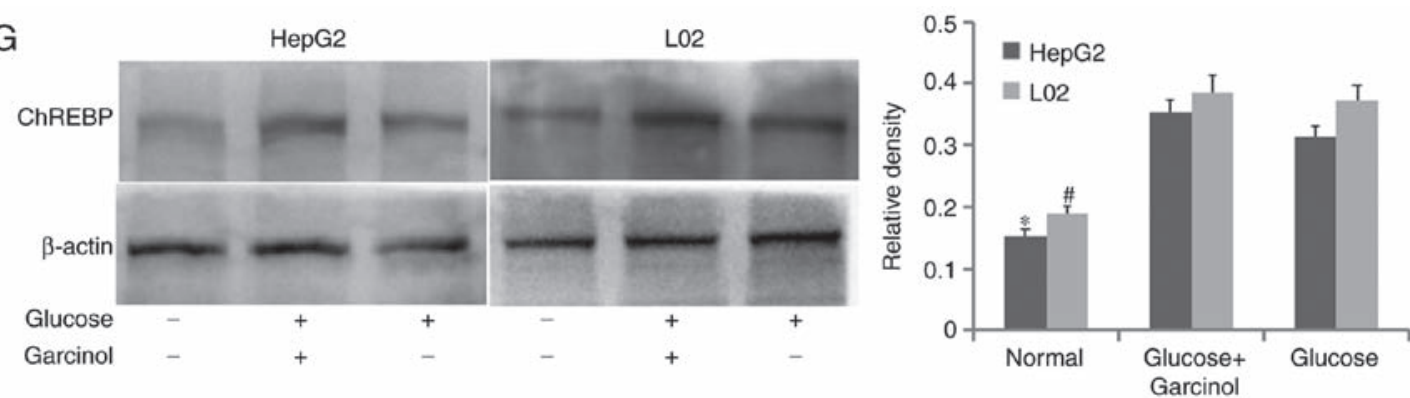

Figure 4. Hepatocellular steatosis is rescued by inhibiting histone acetylation. Chromatin immunoprecipitation assays were performed to measure (A) acetylated H3 and H4 occupancy in the FASN ChoRE, promoter, exon2, and -2 kb regions, and (B) ChREBP-ChoRE binding; values of the normal groups were set as 1 . Total protein and RNA were recovered for (C) western blot analysis and (D) reverse transcription-quantitative polymerase chain reaction analysis of FASN, respectively; $\beta$-actin was used as a loading control. (E) TG contents were measured using a TG assay kit and (F) fat droplets were evaluated using Oil Red O staining. Magnification, x200. (G) Protein levels of ChREBP were determined using western blot analysis; $\beta$-actin was used as a loading control. ${ }^{*} \mathrm{P}<0.05$, vs. glucose+garcinol group in $\mathrm{HepG} 2$; ${ }^{\#} \mathrm{P}<0.05$, vs. glucose+garcinol group in L02. Data are expressed as the mean \pm standard deviation from at least three independent experiments. FASN; fatty acid synthase; TG, triglyceride; ChoRE, carbohydrate response element; ChREBP, ChoRE binding protein. 
the normal group (Fig. 4G). Taken together, these findings that the inhibition of FASN histone acetylation led to no change in the induction of ChREBP but led to a decrease in ChREBP-ChoRE binding, and the subsequent alleviation of TG biosynthesis and lipid accumulation in the HepG2 and L02 cells in response to high glucose stimulation.

\section{Discussion}

In the last decade, as the diets and lifestyles of the Asian population have become rapidly westernized, NAFLD has become one of the most common chronic liver diseases in Asia (31). According to previous studies, diabetes is a high-risk factor for NAFLD $(3,4,32)$. Therefore, the investigation of glucolipid metabolic disorder is particularly important. In the well-recognized 'double-hit' theory, aberrant lipid deposition in hepatocytes, which is also known as hepatic steatosis, is considered to be the pathological basis of NAFLD $(15,16,33)$. FASN, as the key enzyme of de novo lipogenesis, is an important downstream target gene for the conversion of glucose to lipid. In addition, its transcription is closely associated with the development of NAFLD (34-36). In the present study, a significant enhancement of lipogenesis induced by high glucose was observed in HepG2 and L02 cells, as measured by the expression of FASN and lipid content. These pathological changes promote hepatic steatosis and are consistent with a number of previous reports $(9,11,18)$.

Gene expression can be regulated by any of the following control steps: i) chromatin structure; ii) initiation of transcription; iii) processing of the transcript; iv) transport to the cytoplasm; v) translation of mRNA; and vi) stability of protein activity. Among these steps, the chromatin structure is decisive in selective gene expression. Epigenetic phenomena regulate chromatin structure modifications and the initiation of transcription in a manner that alters the availability of genes to the transcription factors required for their expression (37). As structural proteins of eukaryotic chromatin, histones combine with DNA to form the nucleosome, which serves as the basic structural unit of chromatin. Covalent modifications, including acetylation, methylation and phosphorylation, can occur on the amino acid residues in the $\mathrm{N}$-terminal of histones, which are catalyzed by enzymes to alter the chromatin structure, which result in the activation or suppression of gene transcription. Dysregulation of the histone modification of a critical gene has been known to initiate cancer development and contribute to cancer progression (38-41). However, investigations of histone modifications on NAFLD and associated metabolic syndromes remain in their infancy. The present study revealed for the first time, to the best of our knowledge, the association between histone modifications and the expression of FASN in the presence of high glucose and following its withdrawal. It was found that $\mathrm{H} 3$ and $\mathrm{H} 4$ hyper-acetylation, $\mathrm{H} 3 \mathrm{~K} 4$ hyper-trimethylation and H3S10 hyper-phosphorylation within the FASN promoter regions were linked to the activation of FASN and lipogenesis in HepG2 and L02 cells. By contrast, H3K9 and H4K20 hyper-trimethylation were negatively associated with the transcription of FASN in the HepG2 and L02 cells. These findings are consistent with previous studies on histone modifications: Histone acetylation, including $\mathrm{H} 2 \mathrm{~A}, \mathrm{H} 2 \mathrm{~B}, \mathrm{H} 3$ and $\mathrm{H} 4$, is mainly involved in the activation of gene transcription. By contrast, histone methylation and phosphorylation are involved in the activation and suppression of gene transcription according to modification on different residue sites of amino acids. For example, the methylation of H3K4 and phosphorylation of $\mathrm{H} 3 \mathrm{~S} 10$ are involved in gene activation, whereas the methylation of H3K9 and H4K20 leads to the suppression of gene transcription $(25,42-44)$. The phosphorylation of different histones and amino sites is crucial in gene transcription, the condensation status of the chromatin and DNA repair; it is also involved in the regulation of vital activities, including cell growth and division, together with histone acetylation and methylation. At present, reports on histone phosphorylation have mainly focused on serine 10 of H3. In 1991, Hazzalin and Mahadevan (45) found, for the first time, that the phosphorylation of $\mathrm{H} 3$ was correlated with the activity of gene transcription. The phosphorylation of $\mathrm{H} 3$ generated under external stimuli is rapid and transient and can affect phosphorylated $\mathrm{H} 3$ in a different manner from that detected in normal cell division. In addition, the level of this $\mathrm{H} 3$ phosphorylation is positively correlated with the level of acetylation. Numerous studies have confirmed that the phosphorylation of H3S10 induced by various external stimuli involves multiple protein kinase signaling pathways, including mitogen and stress-activated kinase 1, ribosomal protein S6 kinase 2, inhibitor of nuclear factor- $\kappa \mathrm{B}$ kinase- $\alpha$, cAMP-dependent protein kinase C, Fyn and PIM1 (46-48). These kinases are involved in regulating the phosphorylation of H3S10 under different stimuli to induce specific gene transcription.

ChREBP is expressed at high levels in metabolically active organs, including the liver, fat, kidney, small intestine and muscles. Xylulose-5-phosphate, a metabolic product derived from the pentose phosphate signaling pathway, causes the dephosphorylation of ChREBP to promote its nuclear translocation, so that it can be combined with Mlx to form a heterodimer, which finally binds to ChoRE in the promoter region of a target gene to exert the transcriptional activity of ChREBP (5). Global or liver-specific deletion of ChREBP has been found to markedly ameliorate fatty liver disease and improve overall glucose tolerance and insulin sensitivity in ob/ob mice, potentially via a decrease in de novo lipogenesis (13). The overexpression of ChREBP in the liver was reported to increase hepatic steatosis associated with an increased expression of genes regulating fatty acid and TG synthesis in the liver (5). Therefore, ChREBP is a key transcription factor in glucolipid metabolism $(7,10)$. Prior to/when ChREBP initiates the transcription of a target gene, it requires accompanied changes in the local chromatin structure of the gene. ChREBP has been shown to bind in a glucose- and cAMP-dependent manner to ChoREs located in the promoter/enhancer regions of hepatic lipogenic genes, including FASN (49). To further determine the epigenetic mechanisms underlying histone modifications at hepatic FASN promoter regions under high glucose, the present study examined the involvement of ChREBP in FASN histone modifications. The corresponding results indicated that the expression level of ChREBP and the occupancy of ChREBP-ChoRE binding at the FASN promoter were 
positively associated with the expression of FASN during the stimulation of high glucose and following its removal. In addition, the inhibition of ChREBP caused a decline in the levels of ChREBP-ChoRE binding, acetylation of $\mathrm{H} 3$ and $\mathrm{H} 4$, trimethylation of $\mathrm{H} 3 \mathrm{~K} 4$ and phosphorylation of $\mathrm{H} 3 \mathrm{~S} 10$, whereas an increase in the trimethylation levels of $\mathrm{H} 3 \mathrm{~K} 9$ and H4K20 in the FASN promoter regions occurred, leading to the suppression of FASN. In addition, when ChREBP was overexpressed, the levels of ChREBP-ChoRE binding, histone acetylation, trimethylation of $\mathrm{H} 3 \mathrm{~K} 4$ and phosphorylation of H3S10 increased, whereas the modification in the trimethylation levels of $\mathrm{H} 3 \mathrm{~K} 9$ and $\mathrm{H} 4 \mathrm{~K} 20$ decreased within the FASN promoter regions, resulting in high expression levels of FASN, and contributing to increased hepatocellular lipogenesis and hepatic steatosis. Therefore, the present study found that histone modifications in FASN promoter regions were closely associated with ChREBP. Studies have shown that ChREBP accurately regulates the expression of glucose-sensitive genes by forming a complex with transcription factor $\mathrm{p} 300$, coactivator CREBP binding protein (CBP), and RNA polymerase II, and being heterodimerized with Mlx $(50,51)$. However, the components of this complex remain to be fully elucidated. p300 and CBP are proteins functioning as histone-modifying enzymes (52) and are key to open the structure of chromatin. They affect the affinity between histones and DNA by modifying the histone residues, which exposes ChoRE in the promoter of target genes. The heterodimer ChREBP/Mlx of the transcription complex identifies and specifically binds to exposed ChoRE to promote the modification of histone residues by histone-modifying enzymes, and to disassemble the chromatin structure of target genes, eventually leading to the activation of gene transcription. The present study confirmed that glucose affected the transcription of FASN by regulating the expression and transcriptional activity of ChREBP. Furthermore, ChREBP induced FASN ChREBP-ChoRE binding to accelerate the transcription of FASN by facilitating the acetylation of $\mathrm{H} 3$ and $\mathrm{H} 4$, trimethylation of $\mathrm{H} 3 \mathrm{~K} 4$ and phosphorylation of $\mathrm{H} 3 \mathrm{~S} 10$, and inhibiting the trimethylation of $\mathrm{H} 3 \mathrm{~K} 9$ and $\mathrm{H} 4 \mathrm{~K} 20$ in the FASN promoter regions.

As histone modifications are reversible, novel therapeutic strategies to modulate epigenetic aberrancies are being investigated extensively $(38,53)$. Histone acetylation is catalyzed by HAT directly at lysine residues of the N-terminal of histone, enabling local chromatin to be dissected from the histone octamer to facilitate the binding between various transcription factors and DNA regulatory factors, resulting in gene transcription. HDAC decreases the acetylation level of histone to make the association between DNA and the histone octamer closer to further intensify local chromatin contraction and to also inhibit the binding between transcription factors and DNA regulatory factors, which suppress gene transcription. The altered expression and activity of specific histone acetylation-modifying enzymes have been reported to affect phenotypic gene expression in metabolic disease (53). It is known that p300 and CBP are important components of the HAT family members, and garcinol, an organic matter extracted from Indian Garcinia, has the capability to penetrate through cytomembranes to prevent the activity of p300/CBP- and p300/CBP-associated factors $(52,54,55)$. Therefore, the present study used garcinol, as an HAT inhibitor, to further observe the latent impact of histone acetylation on FASN transcription regulated by ChREBP. A previous study showed that glucose, as an endogenous ligand of LXR, upregulated the expression of ChREBP by activating LXR. Glucose was metabolized to xylulose-5-phosphate, which led the dephosphorylation of ChREBP to increase the transcriptional activity of ChREBP, by promoting its translocation from the cytoplasm to the nucleus and its binding to ChoRE in promoters (56). In addition, it has been shown that glucose-activated p300 acetylated ChREBP on Lys672 and increased its transcriptional activity by enhancing its nuclear translocation and recruitment to its target gene promoters, which resulted in increased hepatic lipid synthesis (55). Therefore, the transcriptional activity of ChREBP requires the nuclear translocation and the specific binding of ChREBP-ChoRE in the promoter region of a target gene. The present study found that, when L02 and HepG2 cells were stimulated by a high glucose/garcinol cocktail, garcinol partially antagonized the positive regulation of glucose on the transcriptional activity of ChREBP by inhibiting the effects of p300 and CBP. Therefore, the high glucose/garcinol cocktail reduced ChREBP-ChoRE binding and the acetylation of $\mathrm{H} 3$ and $\mathrm{H} 4$, and caused the downregulated expression of FASN, compared with that following high glucose treatment alone, although the values from the cocktail group were higher than those from the normal group. As garcinol was unable to interfere with the regulation of glucose on the expression of ChREBP, no significant difference was found in the protein level of ChREBP between the cocktail and the high glucose groups, although both were increased compared with that in the normal group. It was concluded that the biological function of ChREBP protein was dependent on its expression level and its transcriptional activity. In conclusion, these findings indicated that the inhibition of histone acetylation under high glucose treatment failed to suppress the expression of ChREBP, however, it inhibited the transcriptional activation of ChREBP by reducing the specific binding of ChREBP-ChoRE in the FASN promoter region, which resulted in the transcriptional repression of FASN and alleviation of hepatocyte fatty degeneration. This process demonstrated that the transcriptional activity of ChREBP is required for the regulation of FASN transcription as histone acetylation leads to conformational transformation in FASN chromatin, indicating that histone acetylation is a crucial mechanism involved in the transcription of FASN modulated by ChREBP.

In conclusion, the results of the present study suggested that ChREBP-mediated histone modifications at the FASN promoter regions in $\mathrm{HepG} 2$ and L02 cells were manifested by $\mathrm{H} 3$ and $\mathrm{H} 4$ hyper-acetylation, H3K4 hyper-trimethylation, H3S10 hyper-phosphorylation and H3K9 and H4K20 hypo-trimethylation, which resulted in an active chromatin structure increasing the transcription of FASN in response to high glucose. Consistent with this finding, the hepatocytes showed enhanced potential for lipogenesis in response to the FASN histone modifications described. Consequently, histone acetylation was provided as a novel theoretical basis for glucolipid metabolism disorder, and for the pathogenesis and therapeutic targets of NAFLD. 


\section{Acknowledgements}

Not applicable.

\section{Funding}

The present study was funded by the National Natural Science Foundation of China (grant nos. 81270494 and 81500443).

\section{Availability of data and material}

The analyzed data sets generated during the study are available from the corresponding author on reasonable request.

\section{Authors' contributions}

$\mathrm{CC}, \mathrm{HY}$ and WS designed the experiments. CC, GH and XY performed the experiments. $\mathrm{CC}, \mathrm{XD}$ and $\mathrm{YZ}$ analyzed the results. CC wrote the manuscript. All the authors read and approved the final manuscript.

\section{Ethics approval and consent to participate}

Not applicable.

\section{Patient consent for publication}

Not applicable.

\section{Competing interests}

The authors confirm that they have no competing interests.

\section{References}

1. Farrell GC and Larter CZ: Nonalcoholic fatty liver disease: From steatosis to cirrhosis. Hepatology 43: S99-S112, 2006.

2. Fan JG and Farrell GC: Epidemiology of non-alcoholic fatty liver disease in China. J Hepatol 50: 204-210, 2009.

3. Ajmera VH, Gunderson EP, VanWagner LB, Lewis CE, Carr JJ and Terrault NA: Gestational diabetes mellitus is strongly associated with non-alcoholic fatty liver disease. Am J Gastroenterol 111: 658-664, 2016.

4. Lomonaco R, Bril F, Portillo-Sanchez P, Ortiz-Lopez C, Orsak B, Biernacki D, Lo M, Suman A, Weber MH and Cusi K: Metabolic impact of nonalcoholic steatohepatitis in obese patients with type 2 diabetes. Diabetes Care 39: 632-638, 2016.

5. Jeong YS, Kim D, Lee YS, Kim HJ, Han JY, Im SS, Chong HK, Kwon JK, Cho YH, Kim WK, et al: Integrated expression profiling and genome-wide analysis of ChREBP targets reveals the dual role for ChREBP in glucose-regulated gene expression. PLoS One 6: e22544, 2011.

6. Shiota M and Magnuson MA: Hepatic glucose sensing: Does flux matter? J Clin Invest 118: 841-844, 2008.

7. Xu X, So JS, Park JG and Lee AH: Transcriptional control of hepatic lipid metabolism by SREBP and ChREBP. Semin Liver Dis 33: 301-311, 2013.

8. Sakiyama H, Wynn RM, Lee WR, Fukasawa M, Mizuguchi H, Gardner KH, Repa JJ and Uyeda K: Regulation of nuclear import/export of carbohydrate response element-binding protein (ChREBP): Interaction of an alpha-helix of ChREBP with the 14-3-3 proteins and regulation by phosphorylation. J Biol Chem 283: 24899-24908, 2008.

9. Uyeda K and Repa JJ: Carbohydrate response element binding protein, ChREBP, atranscription factor coupling hepatic glucose utilization and lipid synthesis. Cell Metab 4: 107-110, 2006
10. Benhamed F, Filhoulaud G, Caron S, Lefebvre P, Staels B and Postic C: O-GlcNAcylation links ChREBP and FXR to glucose-sensing. Front Endocrinol (Lausanne) 5: 230, 2015.

11. Poupeau A and Postic C: Cross-regulation of hepatic glucose metabolism via ChREBP and nuclear receptors. Biochim Biophys Acta 1812: 995-1006, 2011.

12. Denechaud PD, Bossard P, Lobaccaro JM, Millatt L, Staels B, Girard $\mathrm{J}$ and Postic C: ChREBP, but not LXRs, is required for the induction of glucose-regulated genes in mouse liver. J Clin Invest 118: 956-964, 2008.

13. Denechaud PD, Dentin R, Girard J and Postic C: Role of ChREBP in hepatic steatosis and insulin resistance. FEBS Lett 582: 68-73, 2008.

14. Iizuka K: Recent progress on the role of ChREBP in glucose and lipid metabolism. Endocr J 60: 543-555, 2013.

15. Sahini N and Borlak J: Recent insights into the molecular pathophysiology of lipid droplet formation in hepatocytes. Prog Lipid Res 54: 86-112, 2014.

16. Kawano Y and Cohen DE: Mechanisms of hepatic triglyceride accumulation innon-alcoholic fatty liver disease. J Gastroenterol 48: 434-441, 2013.

17. Karagianni $\mathrm{P}$ and Talianidis I: Transcription factor networks regulating hepatic fatty acid metabolism. Biochim Biophys Acta 1851: 2-8, 2015.

18. Wang Y, Viscarra J, Kim SJ and Sul HS: Transcriptional regulation of hepatic lipogenesis. Nat Rev Mol Cell Biol 16: 678-689, 2015.

19. Ozkul Y and Galderisi U: The impact of epigenetics on mesenchymal stem cell biology. J Cell Physiol 231: 2393-2401, 2016.

20. Zoghbi HY and Beaudet AL: Epigenetics and human disease. Cold Spring Harb Perspect Biol 8: a019497, 2016.

21. Martínez JA, Milagro FI, Claycombe KJ and Schalinske KL: Epigenetics in adipose tissue, obesity, weight loss, and diabetes. Adv Nutr 5: 71-81, 2014.

22. Stel J and Legler J: The role of epigenetics in the latent effects of early life exposure to obesogenic endocrine disrupting chemicals. Endocrinology 156: 3466-3472, 2015.

23. Youngson NA and Morris MJ: What obesity research tells us about epigenetic mechanisms. Philos Trans R Soc Lond B Biol Sci 368: 20110337, 2013.

24. Öst A and Pospisilik JA: Epigenetic modulation of metabolic decisions. Curr Opin Cell Biol 33: 88-94, 2015.

25. Lawrence M, Daujat S and Schneider R: Lateral thinking: How histone modifications regulate gene expression. Trends Genet 32: 42-56, 2016.

26. Harr JC, Gonzalez-Sandoval A and Gasser SM: Histones and histone modifications in perinuclear chromatin anchoring: From yeast to man. EMBO Rep 17: 139-155, 2016.

27. Yu H, Wu J, Yang M, Guo J, Zheng L, Peng M, Zhang Q, Xiang Y, Cao J and Shen W: Involvement of liver X receptor alpha in histone modifications across the target fatty acid synthase gene. Lipids 47: 249-257, 2012.

28. Livak KJ and Schmittgen TD: Analysis of relative gene expression data using real-time quantitative PCR and the 2(-delta delta C(T)) method. Methods 25: 402-408, 2001.

29. Rufo C, Teran-Garcia M, Nakamura MT, Koo SH, Towle HC and Clarke SD: Involvement of a unique carbohydrate-responsive factor in the glucose regulation of rat liver fatty-acid synthase gene transcription. J Biol Chem 276: 21969-21975, 2001.

30. Shechter D, Dormann HL, Allis CD and Hake SB: Extraction, purification and analysis of histones. Nat Protoc 2: 1445-1457, 2007.

31. Fan JG, Kim SU and Wong VW: New trends on obesity and NAFLD in Asia. J Hepatol 67: 862-873, 2017.

32. Hazlehurst JM, Woods C, Marjot T, Cobbold JF and Tomlinson JW. Non-alcoholic fatty liver disease and diabetes. Metabolism 65: 1096-1108, 2016.

33. Day CP and James OF: Steatohepatitis: A tale of two 'hits'? Gastroenterology 114: 842-845, 1998.

34. Cordero P, Gomez-Uriz AM, Campion J, Milagro FI and Martinez JA: Dietary supplementation with methyl donors reduces fatty liver and modifies the fatty acid synthase DNA methylation profile in rats fed an obesogenic diet. Genes Nutr 8: 105-113, 2013.

35. Dorn C, Riener MO, Kirovski G, Saugspier M, Steib K, Weiss TS, Gäbele E, Kristiansen G, Hartmann A and Hellerbrand C: Expression of fatty acid synthase in nonalcoholic fatty liver disease. Int J Clin Exp Pathol 3: 505-514, 2010.

36. Jones SF and Infante JR: Molecular pathways: Fatty acid synthase. Clin Cancer Res 21: 5434-5438, 2015. 
37. Kirk H, Cefalu WT, Ribnicky D, Liu Z and Eilertsen KJ: Botanicals as epigenetic modulators for mechanisms contributing to development of metabolic syndrome. Metabolism 57: S16-S23, 2008.

38. Dawson MA and Kouzarides T: Cancer epigenetics: From mechanism to therapy. Cell 150: 12-27, 2012.

39. Tian Y, Wong VW, Chan HL and Cheng AS: Epigenetic regulation of hepatocellular carcinoma in non-alcoholic fatty liver disease. Semin Cancer Biol 23: 471-482, 2013.

40. Gołąbek K, Strzelczyk JK, Wiczkowski A and Michalski M: Potential use of histone deacetylase inhibitors in cancer therapy. Contemp Oncol (Pozn) 19: 436-440, 2015.

41. Yi X, Jiang XJ, Li XY and Jiang DS: Histone methyltransferases: Novel targets for tumor and developmental defects. Am J Transl Res 7: 2159-2175, 2015.

42. Yen CY, Huang HW, Shu CW, Hou MF, Yuan SS, Wang HR, Chang YT, Farooqi AA, Tang JY and Chang HW: DNA methylation, histone acetylation and methylation of epigenetic modifications as a therapeutic approach for cancers. Cancer Lett 373: 185-192, 2016.

43. Su X, Wellen KE and Rabinowitz JD: Metabolic control of methylation and acetylation. Curr Opin Chem Biol 30: 52-60, 2016.

44. Warsito D, Lin Y, Gnirck AC, Sehat B and Larsson O: Nuclearly translocated insulin-like growth factor 1 receptor phosphorylates histone $\mathrm{H} 3$ at tyrosine 41 and induces SNAI2 expression via Brg1 chromatin remodeling protein. Oncotarget 7: 42288-42302, 2016.

45. Hazzalin CA and Mahadevan LC: Dynamic acetylation of all lysine4-methylated histone $\mathrm{H} 3$ in the mouse nucleus: Analysis at c-fos and c-jun. PLoS Biol 3: e393, 2005.

46. Tochiki KK, Maiarú M, Norris C, Hunt SP and Géranton SM: The mitogen and stress-activated protein kinase 1 regulates the rapid epigenetic tagging of dorsal horn neurons and nocifensive behaviour. Pain 157: 2594-2604, 2016.

47. Ho CM, Donovan-Banfield IZ, Tan L, Zhang T, Gray NS and Strang BL: Inhibition of IKK $\alpha$ by BAY61-3606 reveals IKK $\alpha$-dependent histone $\mathrm{H} 3$ phosphorylation in human cytomegalovirus infected cells. PLoS One 11: e0150339, 2016.

48. Zippo A, De Robertis A, Serafini R and Oliviero S: PIM1-dependent phosphorylation of histone $\mathrm{H} 3$ at serine 10 is required for MYC-dependent transcriptional activation and oncogenic transformation. Nat Cell Biol 9: 932-944, 2007.
49. Burke SJ, Collier JJ and Scott DK: cAMP prevents glucose-mediated modifications of histone $\mathrm{H} 3$ and recruitment of the RNA polymerase II holoenzyme to the L-PK gene promoter. J Mol Biol 392: 578-588, 2009.

50. Stoeckman AK, Ma L and Towle HC: Mlx is the functional heteromeric partner of the carbohydrate response element-binding protein in glucose regulation of lipogenic enzyme genes. J Biol Chem 279: 15662-15669, 2004

51. Ma L, Sham YY, Walters KJ and Towle HC: A critical role for the loop region of the basic helix-loop-helix/leucine zipper protein Mlx in DNAbinding and glucose-regulated transcription. Nucleic Acids Res 35: 35-44, 2007.

52. Furdas SD, Kannan S, Sippl W and Jung M: Small molecule inhibitors of histone acetyltransferases as epigenetic tools and drug candidates. Arch Pharm (Weinheim) 345: 7-21, 2012.

53. Knutson SK, Chyla BJ, Amann JM, Bhaskara S, Huppert SS and Hiebert SW: Liver-specific deletion of histone deacetylase 3 disrupts metabolic transcriptional networks. EMBO J 27: 1017-1028, 2008

54. Balasubramanyam K, Altaf M, Varier RA, Swaminathan V, Ravindran A, Sadhale PP and Kundu TK: Polyisoprenylated benzophenone, garcinol, a natural histone acetyltransferase inhibitor, represses chromatin transcription and alters global gene expression. J Biol Chem 279: 33716-33726, 2004.

55. Bricambert J, Miranda J, Benhamed F, Girard J, Postic C and Dentin R: Salt-inducible kinase 2 links transcriptional coactivator p300 phosphorylation to the prevention of ChREBP-dependent hepatic steatosis in mice. J Clin Invest 120: 4316-4331, 2010.

56. He Z, Jiang T, Wang Z, Levi M and Li J: Modulation of carbohydrate response element-binding protein gene expression in 3T3-L1 adipocytes and rat adipose tissue. Am J Physiol Endocrinol Metab 287: E424-E430, 2004.

This work is licensed under a Creative Commons Attribution-NonCommercial-NoDerivatives 4.0 International (CC BY-NC-ND 4.0) License. 